\title{
Análisis crítico de las fuentes \\ estadísticas de consumo alimentario en España. Una perspectiva sociológica ${ }^{1}$
}

\author{
Cecilia Díaz Méndez \\ Universidad de Oviedo \\ cecilia@uniovi.es \\ Paloma Herrera Racionero \\ Universidad Politécnica de Valencia \\ paherra@poli.uned.es \\ Javier Callejo Gallego \\ UNED \\ jcallejo@ poli.uned.es \\ Luis Enrique Alonso Benito \\ Universidad Autónoma de Madrid \\ luis.alonso@uam.es
}

RESUMEN

Este trabajo pretende realizar un análisis crítico de las fuentes estadísticas principales existentes en España sobre alimentación: la Encuesta de Presupuestos Familiares y el Panel de Consumo del Ministerio de Agricultura, Pesca y Alimentación (MAPA). Entendemos que son las limitaciones de estas fuentes las que ofrecen un campo de análisis nuevo para comprender el comportamiento alimentario, su permanencia o cambio. Estas estadísticas proporcionan la posibilidad de conocer los procesos y tendencias del mercado, pero no nos ofrecen un conocimiento global de las bases sociales del hecho alimentario.

Palabras clave: Fuentes Estadísticas, Comportamiento Alimentario, Encuestas de Presupuestos Familiares, Panel de Consumo.

1 Este artículo ha sido elaborado en el marco del proyecto de investigación titulado «Cambio en el consumo alimentario en España: factores sociales que intervienen en la transformación de los comportamientos alimentarios», financiado por el Plan de I+D+I del Principado de Asturias y dirigido por Cecilia Díaz Méndez (Universidad de Oviedo). En él participan, además de los autores de este trabajo, los profesores Ana Jesús López Menéndez, Isabel García Espejo, Marta Ibáñez Pascual (Universidad de Oviedo), Cristóbal Gómez Benito (UNED), Jesús Contreras Hernández y Elena Espeitx Bernat (Universidad de Barcelona) (http://www19.uniovi.es/ecoapli/). 


\section{INTRODUCCIÓN}

La preocupación sociológica por la alimentación es un fenómeno relativamente reciente. Tradicionalmente, el consumo de alimentos ha sido objeto de análisis económico y los aspectos nutricionales de la dieta han propiciado estudios médicos y biológicos, pero ha sido sólo en los últimos años cuando se ha profundizado en la significación social del comportamiento alimentario en las sociedades modernas, ampliando los análisis hacia la comprensión de un comportamiento humano que trasciende lo nutricional y económico, y animando a estudiar los aspectos motivacionales y las variables de tipo social ${ }^{2}$ que puedan explicar las permanencias y los cambios de este comportamiento.

En la actualidad, muchos de estos trabajos ${ }^{3}$ parecen constatar un cambio en el comportamiento alimentario analizado a partir de las transformaciones de una sociedad en proceso de modernización o, para muchos autores, modernización avanzada. El mayor tiempo libre, la urbanización, la incorporación de la mujer al trabajo externo remunerado, o ciertos fenómenos sociales relacionados con la comida (el hambre en el mundo, las enfermedades por productos alimentarios contaminados, los debates sobre las formas de producción, los temas relacionados con la salud y la belleza o el deterioro medioambiental) son factores frecuentemente citados que, aunque no siempre fundamentados, se repiten reiteradamente como explicaciones causales de las modificaciones en el consumo alimentario de los españoles en particular y de los europeos en general. A su vez, son estos mismos factores los que parecen provocar una tendencia homogeneizadora de este comportamiento. Sin embargo, la atención hacia la variedad no suele estar presente en tales análisis. Esta omisión no sólo no ayuda a entender la diversidad de situaciones que estos factores generan, sino que también -y precisamente por ello- les lleva a establecer demasiado apresuradamente ciertas relaciones causales que bien podrían interpretarse de otro modo.

\footnotetext{
2 Esta dimensión sí ha sido tratada desde la antropología, prácticamente desde sus orígenes. Un repaso de la investigación europea sobre alimentación se puede encontrar en Fischler y Garine (1988). En el ámbito español, ver Díaz Méndez y Gómez Benito (2005) y Díaz Méndez (coord.) (2005).

3 En el proyecto de investigación en el que se inserta este artículo se ha realizado una revisión de los estudios sobre alimentación aparecidos en España desde los años ochenta. Algunas de las conclusiones concordantes en la mayoría de los trabajos consultados se resumirían en:
}

— Un aumento de la renta provoca una disminución relativa en el gasto alimentario (ley de Engel), a la vez que ofrece más posibilidades de elección.

— La saturación del mercado hace aumentar la importancia de la calidad y variedad de productos.

- Las actuales circunstancias sociales y culturales están imponiendo cambios sustanciales en los hábitos generales de los consumidores. 
Estos estudios apoyan sus explicaciones en datos cuantitativos procedentes de los registros oficiales (Encuesta de Presupuestos Familiares y Panel de Consumo del MAPA) o de encuestas concretas de carácter privado (Panel Nielsen, Dympanel, Estudios y Encuestas sobre Consumo) ${ }^{4}$. Tales fuentes permiten realizar una clasificación de los aspectos más significativos del cambio en los comportamientos alimentarios en nuestro país. De forma general, las tendencias que ponen de manifiesto nos hacen a los españoles ser cada vez más europeos: nos aproximamos a Europa en el consumo de calorías (entre 3.000 y 3.500 ), en el porcentaje de calorías procedentes de productos animales (40\%), en el porcentaje de gasto destinado a alimentación (20\%) y en el aumento del gasto en comidas fuera del hogar o en productos/servicios ${ }^{5}$. Sin duda, estos datos ofrecen señales para considerar cierta tendencia homogeneizadora; sin embargo, otra de las hipótesis posibles puede considerar que esta tendencia es más visible porque los análisis realizados sobre alimentación la hacen más visible al enfocarla casi exclusivamente, desenfocando u omitiendo otros indicadores que apuntarían hacia las diferencias y variaciones. Si bien es verdad que este tipo de estadísticas constituye una referencia obligada para hablar de los cambios alimentarios en España y para comparar las variaciones alimentarias con las tendencias de otros países, parece necesario preguntarse si estas fuentes están dando respuesta a los interrogantes que, sobre el comportamiento alimentario, se plantean tanto desde el ámbito académico como desde el político o desde los propios consumidores ${ }^{6}$. Es preciso, por ello, afrontar el análisis de las fuentes de referencia y tratar de responder, al menos, a dos interrogantes: en primer lugar, qué información sobre comportamiento alimentario ofrecen estas fuentes y, en segundo lugar, cuáles son sus potencialidades y límites para el estudio de un comportamiento tan complejo.

\section{LAS ENCUESTAS DE PRESUPUESTOS FAMILIARES}

Aunque podemos encontrar precedentes ya en el siglo $\mathrm{XVII}^{7}$, será a mediados del siglo XIX cuando comienzan a desarrollarse en Europa una serie de investigaciones empíricas

\footnotetext{
4 No trataremos aquí estas encuestas, que requerirían de un estudio específico, pues son una referencia muy utilizada por los investigadores (aunque siempre en menor medida que las institucionales aquí analizadas).

5 Con motivo del día mundial de la alimentación, el INE ha editado un boletín informativo especial con cifras básicas sobre alimentación (www.ine.es). Desde el punto de vista sociológico se puede ver Díaz Méndez y Gómez Benito (2005).

6 En este sentido, el registro más utilizado en España para valorar el consumo alimentario, el Panel de Consumo Alimentario del MAPA, cuenta con algunas limitaciones de interés que algunos autores han constatado y que han hecho plantearse si realmente sabemos lo que comemos (Contreras, 1999).

7 William Petty, por ejemplo, realizó un análisis de los gastos de algunas familias obreras inglesas con el propósito de establecer un nuevo sistema de impuestos (Navarro, 1978).
} 
tendentes a conocer la forma de vida en los hogares y cómo gastan las familias sus ingresos. Estas investigaciones, basadas en la recogida estadística de datos sobre el consumo familiar, se centran, en un principio, en el estudio de poblaciones específicas y objetivos muy concretos, ampliándose y sistematizándose hasta constituirse en la mayoría de los países europeos como «Encuesta de Presupuestos Familiares» (Navarro, 1978: 32).

En España, la primera Encuesta de Presupuestos Familiares fue realizada por el Instituto Nacional de Estadística en 1958 con el objetivo de conocer los gastos de consumo de los hogares medios españoles. Como encuestas estructurales o básicas (denominadas abreviadamente EBPF) le siguieron las de 1964-65, 1973-74, 1980-81 y 1990-91, en cuya realización se incorporaron sucesivas mejoras metodológicas ${ }^{8}$.

En 1985 el INE puso en marcha la Encuesta Continua de Presupuestos Familiares (ECPF), una encuesta trimestral realizada a 3.200 hogares (con rotación de 1/8 al trimestre) que amplía el número de rúbricas de bienes y servicios y el número de variables. Desde entonces, las encuestas continuas coexistieron con las básicas hasta 1997, año en que aparece la nueva ECPF tras una reforma metodológica con ajustes en la mecánica de recogida de la información y un incremento del tamaño muestral, permitiendo estimaciones por Comunidades Autónomas. Se introduce también una nueva clasificación de bienes y servicios para adecuarla a las necesidades de la Contabilidad Nacional y facilitar la comparabilidad internacional, especialmente a nivel comunitario, según las demandas de la Oficina de Estadística de la Unión Europea (EUROSTAT) ${ }^{9}$.

Como objetivos esenciales, esta investigación ofrece la lista actualizada de bienes y servicios que componen la cesta de la compra, determinando sus ponderaciones para el cálculo del IPC; proporciona estimaciones del agregado gasto de consumo trimestral y anual para las CC.AA. y su clasificación según diversas variables del hogar o de sus miembros; facilita la estimación, para el conjunto nacional, del consumo anual o trimestral en cantidades físicas de determinados bienes alimenticios; y ofrece anualmente datos estadísticos sobre distintos campos de preocupación social (pobreza y desigualdad, distribución y concentración de la renta y el gasto, equipamiento, vivienda, sanidad, educación...) para posibilitar estudios concretos.

\footnotetext{
8 Aunque de un modo atípico, no se puede negar que ya Le Play, en sus monografías, realizó un resumen del presupuesto anual doméstico de tres familias españolas de 1877-1879. Analizó ingresos y gastos, distribuyéndolos por capítulos presupuestarios para describir la vida doméstica de una familia campesina minera de Lugo, de otra de campesinos de Santander y de una tercera de pescadores de Guipúzcoa (Le Play, 1990). Es, sin duda, una buena referencia histórica para comprender el origen de las actuales encuestas de presupuestos familiares.
}

9 La evolución de las EPF y ECPF se encuentra reflejada en la tabla 1 del Anexo. 
Para cubrir estos objetivos se constituye una base muestral de 8.064 viviendas por trimestre a partir de una exhaustiva lista de variables que incluyen características geográficas, características relativas al hogar y a sus miembros, características relativas a las viviendas y otras características (sanidad, vacaciones, vehículos...). La recogida de información se realiza mediante la anotación directa en una serie de cuestionarios de todos los pagos e ingresos del hogar durante el período muestral, así como información retrospectiva de los pagos efectuados en bienes y servicios cuyo período de referencia es superior al muestral. En total son seis cuestionarios que serán rellenados bien por el entrevistador, bien por la persona responsable de la administración del hogar, bien por todos sus miembros. Se distinguen períodos de colaboración fuerte, trimestre en que la familia anota todos los gastos, y períodos de colaboración débil, en los que se anotan sólo los gastos de referencia superior a la semana.

Los bienes y servicios contemplados en la encuesta están clasificados en 12 grupos. El grupo 01 de la ECPF está destinado a alimentos y bebidas no alcohólicas y aparece subdividido en pan y cereales; carne; pescado; queso, leche y huevos; aceites y grasas; frutas; hortalizas, incluyendo patatas y otros tubérculos; azúcar, confituras, miel, chocolate y confitería; productos alimenticios no comprendidos anteriormente; café, té, cacao; aguas minerales, bebidas gaseosas y zumos; gran compra (compras de productos como alimentos, tabaco, bebidas, artículos de limpieza..., efectuadas con una periodicidad superior a la semana). Estas grandes categorías están subdivididas en 73 registros, sin incluir vinos u otras bebidas alcohólicas, que, a diferencia de lo que sucedía en la ECPF de 1986, se registran en la actualidad en un grupo independiente.

\section{EL PANEL DE CONSUMO DEL MAPA}

El Ministerio de Agricultura, Pesca y Alimentación (MAPA) realiza anualmente desde $1987^{10}$ un seguimiento integral de la alimentación española en el hogar, en la hostelería y en las instituciones mediante el Panel de Consumo Alimentario, y elabora la publicación La alimentación en España, donde se recogen las «principales magnitudes que definen la alimentación de los españoles» (MAPA, 1998: 9). Como objetivo fundamental, esta investigación se propone «conocer la demanda directa de alimentos en el hogar y fuera de él, así como analizar los principales factores que la caracterizan»11 (MAPA, 2004: 32). Se presen-

\footnotetext{
10 Aunque la edición es de 1987, la investigación de referencia se inicia en 1986.

11 Esta definición es común a otros estudios precedentes; sin embargo, los datos más detallados sobre la metodología se han ido ampliando en las sucesivas ediciones. Esta última, la de 2004, es una buena muestra de ello y constituye un texto de referencia básico para recordar la historia de esta investigación.
} 
tan los resultados globales de consumo alimentario de un año concreto, y se ofrecen las cifras de los principales productos que forman la cesta de la compra. Se especifican, además, las variaciones en el volumen de compra, en el precio y en el gasto. Para ello se realiza una encuesta en hogares y otra en hostelería, restauración e instituciones, para posteriormente ordenar y analizar los datos recogidos de acuerdo con una serie de variables analíticas.

Como objetivos concretos, se persigue conocer las cifras de consumo directamente, estudiar la evolución de la dieta alimentaria española, estimar la repercusión de los precios al consumo, obtener información de la importancia relativa de cada forma de comercialización y su evolución, así como de los posibles desajustes entre las producciones obtenidas y la demanda del mercado, con objeto de poder introducir correcciones en los casos que se estime necesario (MAPA, 2000).

Para ello se lleva a cabo una investigación basada en una muestra de 6.000 hogares, 700 establecimientos de hostelería-restauración y 200 establecimientos institucionales, de los que se recoge individualmente información sobre diferentes categorías de alimentos y bebidas a través de un cuestionario. La producción de datos se realiza mediante la colaboración permanente de las mismas familias (la rotación de la muestra aplicada suele ser de un $25 \%$ anual), que en el denominado Diario de Compras registran la cantidad comprada en unidades físicas, el gasto efectuado en dicha compra, el precio unitario del producto (precio de mercado), el lugar de compra y aquellos productos procedentes del autoconsumo o regalo.

Por su parte, los establecimientos de hostelería y restauración e instituciones suministran información de la cantidad comprada en unidades físicas, gasto efectuado, precio unitario y lugar de compra. En este grupo se registran los precios del producto antes de ser ofrecidos a los clientes, es decir, los que paga el responsable del establecimiento.

Los objetivos y la información recogida en esta investigación apenas han variado desde 1986, si bien se han ido introduciendo cambios en la muestra y en las variables analizadas, que son diferentes para los hogares, la hostelería y restauración y las instituciones ${ }^{12}$.

Los alimentos se clasifican en ocho grandes grupos ${ }^{13}$ que incluyen 130 categorías básicas subdivididas en 360 referencias. Estos grandes grupos han sido constantes en el tiempo,

12 Ver evolución en Anexo, tabla 2.

13 Estos grupos son: carnes, pescados y mariscos, leches y derivados lácteos, pan y bollería/pastelería, aceites y grasas vegetales, patatas y hortalizas frescas, frutas y hortalizas, conservas y platos preparados, vinos, bebidas alcohólicas y bebidas analcólicas. 
no así las diferentes categorías en que se han ido desglosando o agrupando en función de la importancia adquirida de los diferentes productos (como, por ejemplo, el desglose de carne fresca - carne congelada, la pizza o la sidra) ${ }^{14}$.

\section{COMPARACIÓN METODOLÓGICA}

Además de las fuentes descritas en los apartados anteriores, existen otras estadísticas sobre consumo alimentario elaboradas por entidades privadas, así como investigaciones relativas a zonas espaciales concretas ${ }^{15} \mathrm{o}$ a problemas específicos ${ }^{16}$. No obstante, la Encuesta Continua de Presupuestos Familiares y el Panel de Consumo del MAPA, por su continuidad, ambición y, sobre todo, por constituir la base empírica fundamental de los estudios que sobre consumo alimentario se realizan en España, se perfilan como las dos principales fuentes estadísticas sobre hábitos alimentarios en general y no sobre un producto o ámbito específico de la alimentación. Ambos estudios han cumplido sobradamente el decenio y metodológicamente utilizan, como veremos, la misma estrategia. Aparentemente, es más lo que les une que lo que les separa, aun cuando sus objetivos son distintos ${ }^{17}$.

\section{Los objetivos: la distancia entre lo que se conoce y lo que se quiere conocer}

La ECPF está destinada a observar los ingresos y gastos de los hogares españoles, sirviendo de base para la elaboración de importantes índices socioeconómicos. Sus objetivos sobrepasan el seguimiento de los gastos relativos a alimentación, constituyendo éstos sólo un capítulo —aun cuando sea uno de los más importantes_- del conjunto del estudio.

Las encuestas del MAPA se acercan más al consumo de alimentos en los hogares, a pesar de que, como repetidamente avisan sus responsables e introductores en las diferentes publicaciones, recogen, a lo sumo, la compra de los mismos. Esto puede ocultar su clara orientación al mercado, concretamente a las distintas modalidades de comercialización y canales de distribución. De hecho, se presentan como un análisis de la demanda, que par-

\footnotetext{
14 Se ha mejorado significativamente la información sobre la metodología del Panel en el último estudio de 2004; además, se precisan las ampliaciones desde 1987 (MAPA, 2004).

15 Éste es el caso de algunos estudios específicos realizados en las Comunidades Autónomas de Madrid, Cataluña, País Vasco o Canarias.

16 Así, existen algunas investigaciones de carácter sociosanitario como el estudio europeo «EPIC»: Dieta, cáncer y salud.

17 Una síntesis de los principales rasgos de cada una de las fuentes tratadas aparece recogida en la tabla 3 del Anexo.
} 
te de lo que la gente consume, cuando, en sentido estricto, más parecen ofrecer un análisis de la demanda a partir de la oferta existente. Habría que hablar, en todo caso, de demanda condicionada. Lo que se recoge, siendo enormemente útil para tal fin, es la demanda que se produce. Al respecto, es sintomático que en ellos se incluya como demanda lo que es regalo (muestras, etc.), es decir, estrategias directas de producción de la demanda. Si es arriesgado establecer una vinculación inmediata entre lo comprado y lo consumido ${ }^{18}$, más lo es entre lo regalado y lo consumido. Por lo tanto, tenemos tres conceptos que quedan frecuentemente confundidos en tales estudios, aun cuando aparezca alguna precaución metodológica en sus introducciones: demanda, consumo y compra. Así, por ejemplo, sólo es registrado el caldo empaquetado y comprado y no el que se hace en casa. Es más, se excluye explícitamente lo que se hace con los alimentos: "No incluya los flanes que usted haga en su casa» (Diario de Compras, Hogares), estando más próxima tal gestión al consumo. El simulacro de caldo o de flan, abstracto y estandarizado, se impone en la contabilidad.

Tales limitaciones para el análisis del consumo se señalan claramente en los estudios MAPA posteriores a 1994. Hay que reconocer que desde que el Ministerio de Agricultura, Pesca y Alimentación adquirió competencias en temas alimentarios se ha ido profundizando y ampliando la recogida de información. En el Panel se miden las cantidades compradas, y no existe la pretensión de analizar comportamientos alimentarios, sino consumos alimentarios, respondiendo a los intereses concretos del organismo en temas de producción y distribución alimentaria. Sin embargo, la ambigüedad presente en algunos de sus análisis suscita dudas sobre su correcta interpretación.

\section{Los instrumentos y su incidencia en las observaciones}

Los dos estudios referidos concretan una forma de las encuestas panel que tiene su lanzamiento a partir de los años sesenta. De las encuestas panel con pocas variables en distintos momentos, se pasa a las que intentan recoger globalmente un comportamiento de manera continuada, sin dejar en manos de la memoria un comportamiento eminentemente estacional. En este sentido, la muestra no sólo está constantemente bajo cuestión, sino, en especial, bajo una cuestión: ha comprado/no ha comprado, distribuida en una considerable variedad y cantidad de productos.

A diferencia de otro tipo de prácticas de observación, al estar constantemente bajo cuestión, las unidades de la muestra han de realizar un esfuerzo constante y continuado. Algo

18 ¿Consumimos, por ejemplo, todo el pan que compramos? Una interesante crítica a la indistinción de «comprado/consumido" se puede ver en Contreras (1999). 
que, por supuesto, no todas las unidades muestrales están dispuestas a llevar a cabo, dándose así la denominada mortandad en la muestra. A este respecto, ha de subrayarse que la literatura metodológica ha hecho más hincapié en las formas de analizar e interpretar los datos de este tipo de encuestas que en la consideración de éstas como una práctica social que lleva al cansancio y reactividad de los observados.

La ventaja de las encuestas panel, al abordar una práctica como la demanda/compra/consumo de alimentos de una manera global y continua, queda ensombrecida por la volatilidad de una muestra pretendidamente sólida. El carácter problemático de la construcción de la muestra se apunta en el estudio que ofrece una información metodológica más transparente, la ECPF, constatando el inusual volumen de incidencias registradas (negativas, ausencias, etc.).

La metodología del Panel del MAPA se ofrece también en las publicaciones periódicas y en la página oficial de la institución. Aparecen datos sobre la muestra y su composición y también sobre la periodicidad y número de los registros realizados, pero no los datos sobre la mortandad de la muestra y su reposición ${ }^{19}$. Las rotaciones son presentadas con escasa precisión: «la normalmente aplicada es del 25\%» (MAPA, 1994) ${ }^{20}$, y las empresas privadas encargadas de su realización no amplían esta información, aunque sí lo hace el MAPA si dispone de ella.

A pesar de que el MAPA ha realizado en los últimos años un importante esfuerzo para mostrar la metodología seguida en la elaboración del Panel y ha ido ampliando progresivamente su muestra, parece que la ECPF ofrece más garantías en cuanto al método de selección, la rotación de la muestra y el tratamiento de la no respuesta.

La muestra del Panel del MAPA no es una constante a lo largo del año y se procesa mensual o trimestralmente (según sea la muestra de hogares o de instituciones y restauración), y, según recoge la metodología publicada, varía en función del grado de colaboración de los hogares y de la calidad de la información requerida. En el caso de la EPF, cada familia de la muestra es investigada cuatro semanas al año (dos de colaboración fuerte y dos débil)21;

19 En el caso del INE, podemos acceder a una detallada información metodológica en la página web (www.ine.es) o en las publicaciones correspondientes. Para el Panel del MAPA existe un resumen metodológico en su web oficial (www.mapya.es) y en la publicación anual en soporte papel La alimentación en España y La alimentación mes a mes.

20 Aunque el Ministerio de Agricultura, Pesca y Alimentación ofrece a los investigadores externos la información disponible siempre que se le solicita, esto no sucede con las empresas privadas encargadas de la realización del Panel.

21 La colaboración fuerte se refiere a la anotación exhaustiva de todos los gastos, la débil a los gastos superiores a la semana. 
la muestra, además, se distribuye uniformemente a lo largo de las semanas del trimestre, de manera que todas ellas estén igual representadas.

En ambos casos, los cuestionarios son minuciosos y cerrados, comprendiendo una gran variedad de alimentos y muchas preguntas sobre cada uno. Lo que es atractivo para el estudioso puede convertirse en una losa para el encargado de completar los impresos, que suele ser casi siempre el ama de casa, por más que los encuestados cuenten con la ayuda remota de un teléfono de consulta gratuita y, en el caso de la EPF, de la visita del agente entrevistador. El Panel ha incorporado desde hace cuatro años un lector óptico que, sin duda, facilita esta tarea.

El orden y contenido de las preguntas tiende a construir un contexto estrechamente relacionado con el mercado, destacando la importancia que se da al registro de la marca de los productos, sin considerar sin embargo otros aspectos de carácter social: salud, quejas, problemas con alimentos, vínculos con asociaciones de consumidores. Por ejemplo, en las preguntas relativas a la leche, en el caso del Panel, ha de registrarse el tipo de envase (brick o pack, términos que ya implican una presunción exclusiva del consumo empaquetado) y se solicita la anotación de la marca. De hecho, se da por supuesto que todo tiene marca, con la excepción de algunos pocos productos para los que se concibe la compra a granel. Así, poco a poco, se van acumulando los ruidos, como la solicitud de la marca de los helados, dejando de lado la posible extensión del consumo de los helados sin marca en muchas partes del territorio español.

En el caso de la EPF, la solicitud de la declaración de ingresos puede llegar a contaminar la declaración del gasto, intensificando la reactividad, las resistencias a ser observado, aminorando tanto los ingresos como los gastos que se registran. Para el encuestado, sus respuestas quedan grabadas para un ente estatal, simbólicamente vinculado con Hacienda y la política impositiva.

Como es bien sabido, la mayor parte de las encuestas continuas panel, en la medida que exigen un esfuerzo, conllevan unas contraprestaciones para quienes colaboran en ella, lo que suele ser ya fuente de crítica en la medida que se abre la posibilidad de sesgar la muestra. Aunque en los estudios analizados no se dice nada al respecto de las mismas, la intuición de que hay contraprestaciones se deriva del tono, en clave de instrucciones o mandatos, de algunos enunciados: "Consejos importantes para colaborar correctamente con Dympanel» (Diario de Compras, Hogares, Dympanel 1997), «Remitir sin falta el lunes día...» (Diario de Compras, Extrahogares, Sigma 1997). La combinación de esta retórica impositiva, que acentúa la percepción de una relación jerárquica entre observador y observado, con la sugerencia de contraprestaciones puede producir distorsiones no desdeñables. 
En los estudios del Instituto Nacional de Estadística, como la ECPF, la sospecha de la contraprestación puede quedar a un lado, pues se establece la obligación de contestar. Pero aquí es la propia imposición de la obligación de declarar ante el INE la que es susceptible de generar una notable reactividad. Hay que tener en cuenta que algunas familias están durante dos años formando parte del panel de la Encuesta de Presupuestos Familiares. En este estudio, se convierte el ama de casa en una especie de agente vicario del INE, tal vez bajo la «sutil» amenaza de sanción en caso de falta de colaboración, como se dice en el propio impreso a rellenar.

\section{¿Recolección o producción de datos?}

La información recopilada en la ECPF, a través de cuestionarios que se rellenan de forma manual, intenta describir todo el contexto del hogar (ingresos percibidos, características, dotaciones, ocupación...) y sus gastos de consumo (adquisición de bienes o servicios, comidas o cenas realizadas en el hogar incluyendo invitados...). Ello exige la colaboración de todos los miembros del hogar de 14 o más años (los de 7 a 13 pueden colaborar voluntariamente), que deben cubrir libretas de gastos individuales. Además, hay una libreta de gastos del hogar a cargo de la persona que lo administra y una ficha del hogar que cubre el encuestador.

Por su parte, el registro del MAPA es realizado por la persona responsable de las compras alimentarias del hogar. Desde el 2000, el MAPA ha facilitado esta tarea por medio de lectores ópticos que, al pasarlos por los códigos de barras de los productos que los posean, registran la información necesaria.

Así, las dos encuestas panel son autoaplicadas. Son los propios encuestados quienes han de completar los registros, a medida que se vayan produciendo las adquisiciones de productos. Requieren anotaciones precisas, exigiéndose cierta familiaridad con la escritura y el manejo de lo escrito. De esta manera, se puede estar privilegiando un determinado perfil social (clases medias urbanas subordinadas, hipercorrectas, de edad media) en detrimento de clases populares, personas mayores y, en general, de todas aquellas personas con actitudes de resistencia al hecho de ser observado o, simplemente, de resistencia a las rutinas, pues el formar parte de la muestra del panel exige la incorporación de una fuerte rutina. 


\section{La construcción de la homogeneidad}

La encuesta mediante cuestionario es, normalmente, una práctica que tiende a privilegiar la observación de la estabilidad, de lo rutinario, legitimado e institucionalizado, produciendo una adscripción con respecto a la norma. En el caso de la ECPF y las encuestas del MAPA, se exige la rutina de la contestación. Así, no son de extrañar conclusiones como la siguiente, con relativa independencia de que ciertamente los hábitos de consumo alimentario cambian lentamente: «En definitiva, parecen confirmarse las apreciaciones realizadas otros años en el sentido de que el gasto alimentario es uno de los criterios más regulares y constantes, dando la impresión de que las amas de casa tuvieran asignado un presupuesto fijo para la cesta de la compra...» (MAPA, 1989: 21). Aunque se trate de una referencia de la década predecente, ¿no será que lo que tienen asignado e incorporado mecánicamente es el procedimiento de respuesta?

Por otra parte, ambas fuentes posibilitan comparaciones regionales, si bien en el caso del Panel del MAPA la distribución regional no tiene en cuenta las CC.AA. sino que se realiza agrupando autonomías con afinidades geográficas y gastronómicas que el propio Ministerio elabora ${ }^{22}$. En el año 2000 ya se incluyen cifras por Comunidades Autónomas, excluyendo Ceuta y Melilla. No deja de ser significativo que ambas exclusiones sean también presumiblemente dos claras excepciones a la reiterada homogeneidad atribuida al comportamiento alimentario, que en estos dos casos está marcado por los hábitos característicos de su numerosa población musulmana. No obstante, el coste de la ampliación de la muestra a estos territorios puede justificar su no inclusión.

En el mismo sentido actuaría el concepto de hogar con el que trabajan ambas fuentes. En ellas se define hogar como «persona o conjunto de personas que ocupan en común una vivienda familiar principal o parte de ella y consumen y/o comparten alimentos y otros bienes con cargo a un mismo presupuesto». Esta definición excluye casos que podrían ser fuente de variedad desde el punto de vista de la organización y el consumo alimentario. En concreto, en la ECPF se hace constar expresamente que no se consideran hogares los pisos compartidos por estudiantes, ya que éstos dependen del presupuesto de sus respectivas familias. Cabría preguntarse, además, si el hogar es la unidad más adecuada desde el punto de vista de los consumos y los comportamientos alimentarios. No es extraño encontrar personas que «comparten los alimentos» sin participar «en un mismo presupuesto» (como los hijos, emancipados, que comen diariamente en casa de sus padres).

22 Desde 1987 el Panel cuenta con datos por CC.AA. que han ido apareciendo publicados a partir del año 2000. Para su recogida se emplea la zonificación geográfica del propio Ministerio y una muestra aproporcional, según aparece en la metodología de la web del MAPA, para garantizar un mínimo de 150 hogares por territorio. 
Una de las diferencias más claras entre las dos fuentes es la relativa al tratamiento del consumo alimentario fuera del hogar. Los registros del MAPA permiten el análisis del consumo en hostelería y restauración, con un universo nacional semejante al de los hogares, y también ofrecen la posibilidad de realizar análisis del consumo institucional, si bien éste no se diferencia por regiones ni áreas geográficas. Por su parte, la ECPF nos permite analizar la frecuencia y el gasto del consumo alimentario fuera del hogar a partir de indicadores como comidas y cenas en restaurantes; gastos en comidas en otras viviendas familiares distintas a la residencia del hogar seleccionado; cafés, bares y similares; gastos en platos preparados para llevar; comedores de empresa y oficinas públicas; comedores escolares; comedores universitarios, etc. Este tipo de información no está contemplada en el Panel, que, aunque ofrece información sobre el gasto y el tipo de productos adquiridos por los restaurantes y las instituciones, no se pone en relación con el gasto de los hogares en ellos ${ }^{23}$.

Por último, el análisis del contenido nutricional aparece recogido únicamente en el registro del MAPA, que incorpora tablas de contenido nutricional de los productos y también analiza la dieta de los hogares comparándola con los patrones de la dieta mediterránea. Asimismo, este estudio realiza un análisis comparativo de la dieta entre regiones. Ahora bien, esta dieta - y las comparaciones subsiguientes - se cuantifica solamente a partir de los productos "crudos», cuando es bien sabido que los valores nutricionales se alteran profundamente en los procesos de cocción, fritura, asado... a los que los diferentes estilos culinarios, tanto familiares como regionales, someten a los alimentos. Una vez más, la homogeneidad percibida es una homogeneidad más puesta que encontrada.

Debemos reconocer que el organismo que más ha avanzado en el estudio sobre la alimentación en España ha sido el Ministerio de Agricultura, Pesca y Alimentación. Conocedor de las limitaciones del Panel para captar en su totalidad el comportamiento alimentario, y también consciente de ser el referente institucional para la investigación alimentaria, ha promovido diversos trabajos que complementan y amplían la información obtenida con el Panel: El Observatorio del consumo y la distribución alimentaria ${ }^{24}$ y el Barómetro del clima de confianza del sector agroalimentario ${ }^{25}$ constituyen un nuevo referente para este campo de análisis. EI MAPA se ha lanzado también a explorar hábitos de consumo novedosos, como lo muestra el reciente estudio sobre los hábitos alimentarios de los inmigrantes (MAPA, 2004).

\footnotetext{
23 Las limitaciones para el estudio del consumo fuera del hogar a partir de los datos del Panel del MAPA las podemos encontrar en Rama (1997).

24 Analiza trimestralmente datos de consumo y compra con metodología cuantitativa y cualitativa.

25 Es un estudio sobre la coyuntura del sector agroalimentario español, con análisis sobre las percepciones de los consumidores y sus hábitos de compra.
} 


\section{CONCLUSIÓN}

Los dos estudios analizados aquí no sólo son importantes, sino también referencia obligada para toda investigación sobre la evolución del consumo de alimentos en España. Su utilidad, además, está avalada tanto por la gran cantidad de información que suministran como por el uso que hacen de ella los investigadores para estudiar el comportamiento alimentario. Ahora bien, ni sus datos ni sus conclusiones pueden tomarse, como a menudo se hace, como fuentes de datos primarios. Las categorizaciones y métodos por medio de los cuales hemos visto que se elaboran esos datos y conclusiones los cargan con importantes sesgos incorporados.

La relevancia de reflexiones como las que acabamos de realizar aquí, en las que se destacan algunas de las características de fuentes que son de uso frecuente en el ámbito académico, apunta al reconocimiento de sus límites. La realidad se analiza con frecuencia a partir de los datos ofrecidos por este instrumental metodológico. Reconocer sus limitaciones permite aproximarse con cautela a un mundo que es, en sí mismo, complejo y diverso, y que se resiste a ser cuantificado y clasificado. Perder esto de vista puede distorsionar un adecuado estudio de la realidad alimentaria y cerrar las posibilidades de continuar investigando hechos y prácticas sobre los que aún queda mucho por conocer. 


\section{ANEXO}

TABLA

\section{Evolución de las Encuestas de Presupuestos Familiares (INE)}

\begin{tabular}{|c|c|c|c|c|}
\hline & Años & Características & Muestra & \\
\hline \multirow[t]{5}{*}{$\begin{array}{l}\text { Encuestas } \\
\text { Estructurales }\end{array}$} & 1958 & $\begin{array}{l}\text { - Primera encuesta por muestreo realizada por } \\
\text { el INE } \\
\text { - Nivel de desagregación de los gastos: } 68 \text { rú- } \\
\text { bricas }\end{array}$ & $\begin{array}{l}\text { Sólo «hogares } \\
\text { medios } \\
\text { españoles»: } \\
3.857 \text { hogares }\end{array}$ & $\begin{array}{l}\text { Trabajos de } \\
\text { campo: mes } \\
\text { de marzo }\end{array}$ \\
\hline & $1964-65$ & $\begin{array}{l}\text { - Cubre todo el ámbito poblacional } \\
\text { - Nivel de desagregación de los gastos: } 90 \\
\text { - Se registran por primera vez ingresos desagre- } \\
\text { gados }\end{array}$ & Ámbito nacional & $\begin{array}{l}\text { Trabajo de } \\
\text { campo: } 1 \text { año }\end{array}$ \\
\hline & $1973-74$ & $\begin{array}{l}\text { - Estudia gastos, ingresos y ahorro } \\
\text { - Aumenta el nivel de desagregación de los gastos } \\
\text { - Diseño muestral como la Encuesta General de } \\
\text { Población }\end{array}$ & Ámbito nacional & $\begin{array}{l}\text { Trabajo de } \\
\text { campo: } 1 \text { año }\end{array}$ \\
\hline & $1980-81$ & $\begin{array}{l}\text { - Estudia gastos, ingresos y ahorro } \\
\text { - Incorpora módulos de condiciones de vida } \\
\text { (equipamiento del hogar, características de la } \\
\text { vivienda, nivel de estudios, cobertura sanitaria) } \\
\text { - Aumenta el nivel de desagregación de los gas- } \\
\text { tos (630) }\end{array}$ & Ámbito nacional & $\begin{array}{l}\text { Trabajo de } \\
\text { campo: } 1 \text { año }\end{array}$ \\
\hline & $1990-91$ & $\begin{array}{l}\text { - Última encuesta estructural } \\
\text { - Estudia gastos, ingresos, préstamos y amorti- } \\
\text { zaciones. Inversión en vivienda } \\
\text { - Intensificación de características demográficas } \\
\text { y sociales de los miembros del hogar } \\
\text { - Aumento de desagregación de gastos (900) e } \\
\text { ingresos } \\
\text { - Introducción de un módulo sobre pobreza sub- } \\
\text { jetiva }\end{array}$ & $\begin{array}{l}\text { Ámbito nacional: } \\
3.200 \text { hogares } \\
\text { por trimestre }\end{array}$ & $\begin{array}{l}\text { Trabajo de } \\
\text { campo: } 1 \text { año }\end{array}$ \\
\hline \multirow[t]{2}{*}{$\begin{array}{l}\text { Encuesta } \\
\text { Continua } \\
\text { Presupuestos } \\
\text { Familiares }\end{array}$} & 1985 & $\begin{array}{l}\text { - Estudia gastos de consumo (anual y trimestral) } \\
\text { e ingresos } \\
\text { - Aumento de rúbricas de bienes y servicios } \\
\text { (400) } \\
\text { - Introduce nuevas preguntas acerca de ingre- } \\
\text { sos, viviendas y características del sustentador } \\
\text { principal }\end{array}$ & $\begin{array}{l}\text { Ámbito nacional: } \\
3.200 \text { hogares } \\
\text { por trimestre }\end{array}$ & $\begin{array}{l}\text { Panel rotatorio } \\
\text { trimestral }\end{array}$ \\
\hline & 1997 & $\begin{array}{l}\text { - Estudia gastos de consumo (anual y trimestral) } \\
\text { e ingresos } \\
\text { - Rúbricas de bienes y servicios (400) } \\
\text { - Reforma metodológica a partir del tercer trimes- } \\
\text { tre de } 1997 \\
\text { - Nueva clasificación de bienes y servicios, más } \\
\text { adecuada a las necesidades de la Contabilidad } \\
\text { Nacional y a los requerimientos de la Oficina de } \\
\text { Estadística de la Unión Europea (EUROSTAT) }\end{array}$ & $\begin{array}{l}\text { Ámbito nacional: } \\
8.064 \text { viviendas } \\
\text { por trimestre }\end{array}$ & $\begin{array}{l}\text { Panel rotatorio } \\
\text { trimestral }\end{array}$ \\
\hline
\end{tabular}


TABLA 2

\section{Evolución Panel de Consumo (MAPA)}

\begin{tabular}{|c|c|c|c|}
\hline Años & $\begin{array}{r}\text { Criterios } \\
\text { de desglose }\end{array}$ & $\begin{array}{r}\text { Tamaño } \\
\text { de la muestra }\end{array}$ & $\begin{array}{r}\text { Consumo } \\
\text { extradoméstico }\end{array}$ \\
\hline
\end{tabular}

\begin{tabular}{llll}
\hline 1987-1988 & Geográficos & 2.500 hogares & 500 establecimientos \\
Empresa & Tamaño del hábitat & & $\begin{array}{l}\text { Recogida información } \\
\text { dos veces al año }\end{array}$ \\
IOPE-ETMAR & $\begin{array}{l}\text { Clases sociales } \\
\text { Lugar de compra }\end{array}$ & & \\
\hline
\end{tabular}

\begin{tabular}{|c|c|c|c|c|}
\hline \multirow{3}{*}{$\begin{array}{l}\text { Sep. } 1989 \\
\text { Empresa } \\
\text { IOPE-ETMAR }\end{array}$} & Geográficos & 2.500 hogares & 500 establecimientos & Recogida información \\
\hline & $\begin{array}{l}\text { Tamaño del hábitat } \\
\text { Clases sociales }\end{array}$ & & hostelería & dos veces al año \\
\hline & $\begin{array}{l}\text { Edad ama de casa } \\
\text { Tamaño familia } \\
\text { Lugar de compra }\end{array}$ & & $\begin{array}{l}100 \text { establecimientos } \\
\text { institucionales }\end{array}$ & $\begin{array}{l}\text { Recogida información } \\
\text { una vez al año }\end{array}$ \\
\hline
\end{tabular}

\begin{tabular}{|c|c|c|c|c|}
\hline $\begin{array}{l}\text { Sep. } 1992 \\
\text { Empresa } \\
\text { Nielsen }\end{array}$ & $\begin{array}{l}\text { Los anteriores, } \\
\text { añadiendo actividad } \\
\text { fuera del hogar } \\
\text { del ama de casa }\end{array}$ & 2.500 hogares & $\begin{array}{l}500 \text { establecimientos } \\
\text { hostelería } \\
100 \text { establecimientos } \\
\text { institucionales }\end{array}$ & $\begin{array}{l}\text { Recogida información } \\
\text { dos veces al año } \\
\text { Recogida información } \\
\text { una vez al año }\end{array}$ \\
\hline $\begin{array}{l}\text { Enero } 1993 \\
\text { Empresa } \\
\text { Nielsen }\end{array}$ & $\begin{array}{l}\text { Los mismos criterios } \\
\text { de desglose que en } \\
\text { años anteriores }\end{array}$ & 2.000 hogares & $\begin{array}{l}500 \text { establecimientos } \\
\text { hostelería } \\
100 \text { establecimientos } \\
\text { institucionales }\end{array}$ & $\begin{array}{l}\text { Recogida información } \\
\text { dos veces al año } \\
\text { Recogida información } \\
\text { una vez al año }\end{array}$ \\
\hline $\begin{array}{l}\text { 1994-Sep. } 1996 \\
\text { Empresa } \\
\text { Nielsen }\end{array}$ & $\begin{array}{l}\text { Los mismos criterios } \\
\text { de desglose que en } \\
\text { años anteriores }\end{array}$ & 2.500 hogares & $\begin{array}{l}500 \text { establecimientos } \\
\text { hostelería } \\
100 \text { establecimientos } \\
\text { institucionales }\end{array}$ & $\begin{array}{l}\text { Recogida información } \\
\text { dos veces al año } \\
\text { Recogida información } \\
\text { una vez al año }\end{array}$ \\
\hline $\begin{array}{l}\text { Enero 1997- } \\
\text { Dic. } 2000 \\
\text { Empresas } \\
\text { Dympanel, } \\
\text { Mercasa } \\
\text { y Sigma2 }\end{array}$ & $\begin{array}{l}\text { Se introduce un nuevo } \\
\text { criterio: la presencia } \\
\text { de niños en el hogar }\end{array}$ & 5.400 hogares & $\begin{array}{l}700 \text { establecimientos } \\
\text { hostelería } \\
200 \text { establecimientos } \\
\text { Institucionales }\end{array}$ & $\begin{array}{l}\text { Recogida información } \\
\text { cuatro veces al año }\end{array}$ \\
\hline
\end{tabular}

\begin{tabular}{|c|c|c|c|c|}
\hline $\begin{array}{l}2001 \\
\text { Empresas }\end{array}$ & $\begin{array}{l}\text { Mismos criterios que } \\
\text { en el período anterior }\end{array}$ & 6.000 hogares & $\begin{array}{l}700 \text { establecimientos } \\
\text { hostelería }\end{array}$ & $\begin{array}{l}\text { Recogida información } \\
\text { cuatro veces al año }\end{array}$ \\
\hline \multicolumn{5}{|l|}{ Dympanel, } \\
\hline Mercasa & & & 200 establecimientos & \\
\hline y Sigma2 & & & institucionales & \\
\hline
\end{tabular}

FUENTE:

La alimentación en España, MAPA, 2000, p. 23, y elaboración propia. 


\section{Análisis comparativo de la EPF y el Panel del MAPA}

Encuestas de Presupuestos Familiares (INE)

La alimentación en España (MAPA)

Series

históricas

disponibles
EBPF: 1958, 1964-65, 1967-68, 1973-74, 1980-

1981, 1990-91.

ECPF: 1985, 1997 (trimestral)
Objetivos Estimación del gasto agregado de consumo trimestral por y su clasificación para diversas variables del hogar.

Estimación del cambio interanual trimestral del gasto de consumo.

Obtención del agregado gasto anual como suma de las estimaciones trimestrales a nivel de Comunidad Autónoma.

Estimación para el conjunto nacional del consumo anual y trimestral en cantidades físicas de determinados artículos alimenticios.

Construcción de indicadores sobre distintos campos de preocupación social como pobreza y desigualdad.
Conocer cifras de consumo.

Estudio de la evolución de la dieta alimentaria española.

Estimación de la repercusión de los precios en el consumo.

Información de la importancia relativa de cada forma de comercialización y su evolución.

Análisis de los posibles desajustes entre las producciones obtenidas y la demanda del mercado, con objeto de poder introducir correcciones, en los casos que se estime necesario.

Se consideran todos los hogares de la península, Baleares y Canarias. Es «hogar» toda persona o conjunto de personas que ocupan en común una vivienda familiar o parte de ella y consumen alimentos y otros bienes con cargo a un mismo presupuesto.

Se incluye también una investigación en hostelería y otra en instituciones.

La muestra útil actual ha pasado de ser 2.500 hogares hasta 1996 a 5.400 en 1997.

La muestra se distribuye respetando la distribución de población, pero favoreciendo la proporción de los estratos más pequeños para que mejore su representatividad. de cada Comunidad Autónoma.

Renovación La muestra se distribuye uniformemente a lo larde la muestra go de las semanas del trimestre para que todas ellas estén igualmente representadas. Cada vivienda permanece en la muestra ocho trimestres consecutivos, siendo cuatro de colaboración fuerte $(G)$ y otros cuatro de colaboración débil (g), según el siguiente esquema: GG gg GG gg.
La muestra no es una constante a lo largo del año, fluctuando según el grado de colaboración de los hogares (si la información recogida en un momento dado no supera los controles de calidad requeridos, ese hogar se desecha en ese período de tiempo y se utiliza un sustituto).

La colaboración oscila, en media, entre uno y dos años; la rotación de la muestra aplicada es de un $25 \%$ anual. 
TABLA 3

\section{Continuación}

Metodología La encuesta ha sido objeto de una reforma metodológica a partir del tercer trimestre de 1997, con ajustes en la mecánica de recogida y un incremento del tamaño muestral, para realizar estimaciones por CC.AA. Al mismo tiempo, ha sido introducida una nueva clasificación de bienes y servicios conforme a la cual se codifican los diferentes gastos efectuados por los hogares, con el fin de hacerla más adecuada a las necesidades de la Contabilidad Nacional y para facilitar la comparabilidad internacional, según los requerimientos de EUROSTAT.

La adaptación metodológica del nuevo proyecto ha supuesto también algunos cambios sustanciales en los criterios para registrar determinadas partidas de gasto y en los períodos de referencia de la información.

Recogida La recogida de datos se realiza proporcionando a de datos los hogares cuestionarios específicos en los que deben anotar de forma continua los gastos realizados. Al recoger los datos, el encuestador completa la información con entrevista directa.
La metodología del Panel de Consumidores, aunque sencilla en su concepción, es complicada en su aplicación, debido a la magnitud de la muestra que se maneja y a la diversidad de variables del cuestionario que se ha de cumplimentar diariamente. Además, al tratarse de una muestra casi permanente, necesita de un índice de captaciones perdurables en el tiempo y de un equipo de recogida de datos altamente cualificado.

En los años 1992 y 1996 hubo un período de tres meses, de octubre a diciembre, no cubierto por el Panel de Hogares, Hostelería y Restauración.

En 1993 y en 1997 se reorganizó el estudio y se renovó totalmente la muestra, manteniéndose la metodología.

La recogida de los datos de consumo de los hogares se realiza mediante panel, es decir, con la colaboración permanente de las mismas familias durante todos los días del año, cumplimentando los cuestionarios, rasgo que proporciona una mayor calidad y rigor a la información. Desde el año 2000 se realiza con lector óptico de código de barras.

Cantidad comprada en unidades físicas, gasto efectuado en dicha compra, precio unitario, lugar de compra (salvo investigación institucional).

Zonas geográficas, nivel socioeconómico del hogar, tamaño del hábitat, número de miembros del hogar, edad del responsable de la compra, actividad del ama de casa, presencia de niños y edad de los niños, tipo de establecimiento donde se compra.

Clases según nivel de instrucción y responsabilidad en el trabajo.

Baja.

Media-Baja.

Media.

Alta. 


\section{REFERENCIAS BIBLIOGRÁFICAS}

CONTRERAS, J. (1993): Antropología de la alimentación, EUDEMA, Madrid.

- (1999): «Estadísticas y pautas de consumo: ¿sabemos realmente lo que comemos?», en La Alimentación Mediterránea, Institut Català de la Mediterrania, Ed. Icaria, Barcelona.

DÍAZ MÉNDEZ, C. (coord.) (2005): ¿Cómo comemos? Cambios en los comportamientos alimentarios de los españoles, Fundamentos, Madrid.

DÍAZ MÉNDEZ, C., y GÓMEZ BENITO, C. (2005): Consumo, seguridad alimentaria y salud, número monográfico de la Revista Internacional de Sociología, CIS, Madrid.

- (2005): «El cambio alimentario en España», en Atlas de la España rural, MAPA, Madrid.

FISCHLER, C., y GARINE, I. (1988): «Ciencias humanas y alimentación: tendencias actuales en la investigación europea», en Carencia alimentaria. Una perspectiva antropológica, Serbal-Unesco, París, pp. 65-87.

INE (1986): Encuesta Continua de Presupuestos Familiares, base 1985. Metodología.

- (1998): Encuesta Continua de Presupuestos Familiares, base 1997. Metodología.

- (varios años): Encuesta Continua de Presupuestos Familiares. Análisis de la falta de respuesta (www.ine.es).

LE PLAY, F. (1990): Campesinos y pescadores del norte de España, MAPA, Clásicos Agrarios, Madrid.

MAPA (varios años): La alimentación en España (www.mapya.es).

- (2004): La alimentación en España, MAPA, Madrid.

- (2004): Hábitos alimentarios de los inmigrantes en España, MAPA y A. C. Nielsen, Madrid.

NAVARRO, M. (dir.) (1978): La sociedad de consumo y su futuro. El caso de España, INE, Madrid.

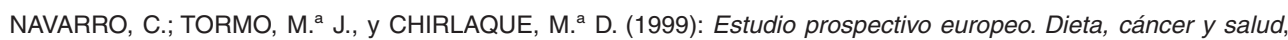
Consejería de Sanidad y Consumo, Dirección General de Salud Pública de Murcia.

RAMA, R. (1997): «Evolución y características de la alimentación fuera del hogar y del consumo de alimentos procesados en España», Agricultura y Sociedad, n. ${ }^{\circ}$ 84, pp. 107-140.

REBOLLO, A. (2001): La estructura del consumo en España, Ministerio de Sanidad y Consumo. 


\section{ABSTRACT}

This work aims at making a critical analysis of the main statistical sources existing in Spain regarding food: the «Encuesta de Presupuestos Familiares y el Panel de Consumo del Ministerio de Agricultura, Pesca y Alimentación» (MAPA) (Survey of Family Budgets and the Consumer Panel of the Ministry of Agriculture, Fisheries and Food). We understand that it is the limitations of these sources that offer a new field of analysis for understanding conduct regarding food, its permanence or change. These statistics provide us with the possibility of learning about market processes and trends but do not offer us a comprehensive knowledge of the social bases of food facts.

Key words: Statistical Sources, Conduct Regarding Food, Surveys of Family Budgets, Consumer Panel. 